\title{
L'écriture, le trauma et la chair
}

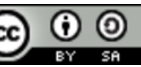

Fred Poché *

Université Catholique del'Ouest

Angers, Francia

Para citar este artículo: Poché, Fred. «L'écriture, le trauma et la chair». Franciscanum 177, Vol. 64 (2022): 1-20.

«L'ennui c'est qu'il y ait des corps et plus encore des sexes ${ }^{1}$.

L. Althusser

«La circoncision, je n'ai jamais parlé que de ça $»^{2}$.

J. Derrida

\section{Résumé}

La présente contribution propose d'étudier la façon dont s'articulent l'histoire singulière d'un philosophe et sa pensée, en nous arrêtant, plus particulièrement, sur deux auteurs d'envergure : Louis Althusser et Jacques Derrida. Ceux-ci, en effet, évoqueront, dans leur autobiographie, des blessures personnelles, voire des traumatismes. À de nombreuses reprises, le premier fut hospitalisé en psychiatrie et le second aborda, très fréquemment, la question la circoncision. Dans la présente étude, il s'agira, en premier lieu, d'appréhender l'articulation entre le nom et le processus de subjectivation au regard de ces deux philosophes d'origine algérienne. Ensuite, l'on s'efforcera de prendre au sérieux la question du trauma afin d'analyser ses effets sur leur pensée. Enfin, en s'appuyant sur leurs productions, l'on regardera dans quelle mesure le « réel » abordé par les deux philosophes fait écho à ce « réel » qui s'invita par effraction dans leur existence.

\section{Mots-clés}

Déconstruction, idéologie, pensée, réel, trauma.

\footnotetext{
* Doctor en filosofía por la Universidad París X-Nanterre, tiene un grado en filosofía por el Instituto Católico de París, un postgrado en Ciencias del lenguaje de la Sorbonne Nouvelle, así como la acreditación otorgada por la Universidad de Estrasburgo como supervisor de estudiantes de doctorado. Actualmente es profesor de filosofía contemporánea en la Faculté des Sciences Humaines et Sociale, de la Université catholique de l'Ouest, en Francia. Es miembro de RPpsy, «Recherches en Psychopathologie et psychanalyse», en Angers, Francia. ORCID: https://orcid.org/0000-0003-0441-2494. Contacto: fred.poche@ wanadoo.fr.

${ }^{1}$ L. Althusser, L'avenir dure longtemps (Paris: Stock/IMEC, 1992), 31.

2 J. Derrida, «Circonfession», in Jacques Derrida par G. Bennington et Jacques Derrida (Paris: Seuil, 1999), 70.
} 


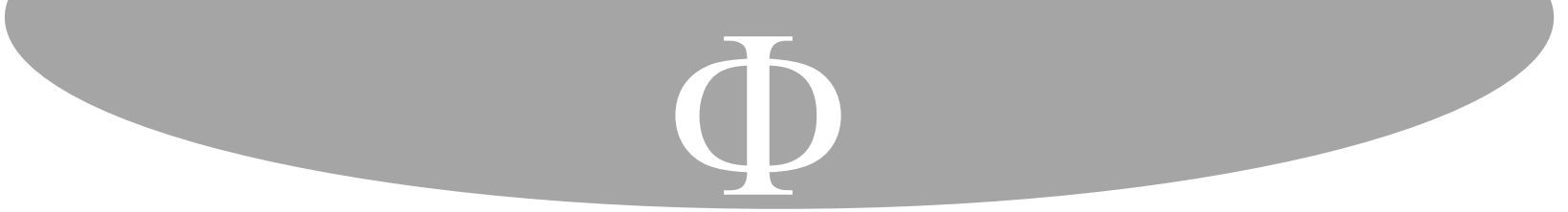

Notre auteur se définissait donc comme une sorte de marrane de la culture catholique française. Il se comparera d'ailleurs à ces marranes qui ne se disent même pas Juifs dans le secret de leur cœur. Il soulignera pourtant qu'il n' aurait «jamais touché à son nom....» ${ }^{14}$. La question de l'appellation se retrouve chez Derrida également lorsqu'il aborde la question de la judéité. Le philosophe précisera à ce propos n'avoir jamais entendu le mot « juif » dans sa famille, même comme une désignation neutre et destinée à classer ; et encore moins comme un nom permettant d'identifier l'appartenance à une communauté sociale, ethnique ou religieuse. Il lui semblera l'avoir entendu à l'école d'El Biar déjà sémantiquement chargé par l'insulte et associé à une blessure, une injustice, un déni de droit; mais jamais comme l'expression du droit d'appartenir à un groupe légitime. Avant d'y comprendre quoi que ce soit, le philosophe reçut ce mot comme un coup, comme une dénonciation, une délégitimation précédant tout droit $^{15}$.

Cette question du nom, et corrélativement de l'identité, travaille constamment la pensée de Derrida. «Le nom, demande-t-il d'ailleurs, qu'appelle-on ainsi ? Qu'entend-on sous le nom de nom ? Et qu'arrive-t-il quand on donne un nom ? Que donne-t-on alors ? On n'offre pas une chose, on ne livre rien et pourtant quelque chose advient qui revient à donner, comme l'avait dit Plotin du Bien, ce qu'on n'a pas. Que se passe-t-il surtout quand il faut surnommer, re-nommant là où justement, le nom vient à manquer ?» ${ }^{16}$.

Incontestablement, l'histoire de son ami, Louis Althusser, entre en résonance avec ces questions ${ }^{17}$. À l'occasion du décès de l'auteur de Pour Marx, le penseur de la déconstruction déclarera, d'ailleurs : «... nous ne sommes jamais nous-mêmes que depuis ce lieu de résonance de l'autre ${ }^{18}$. Pourtant, ce dit «lieu de résonance» revêt parfois la forme d'une camisole qui étouffe le sujet et l'empêche d'exister vraiment, de faire $s a$ vie.

Dans L'avenir dure longtemps, ouvrage publié après le meurtre de son épouse, et dont il dira qu'il constitue sa traumabiographie ${ }^{19}$, Althusser donne des informations essentielles, lui aussi, sur l'attribution de son prénom. Dans sa jeunesse, sa mère très amoureuse d'un homme apprit, un jour, pendant la guerre, que celui-ci venait de décéder. Son frère qui lui

\footnotetext{
${ }^{14}$ J. Derrida, «Une folie doit veiller sur la pensée», entretien avec François Ewald, Magazine Littéraire 286 (1991), 20.

15 J. Derrida, «Abraham, l'autre», Judéités. Questions pour Jacques Derrida (Paris: Galilée, 2003), 19.

16 J. Derrida, «Prière d'insérer», in Sauf le nom (Paris: Galilée, 1993), 1. Cf. F. Poché, Penser avec Jacques Derrida. Comprendre la déconstruction (Lyon: Chronique sociale, 2007). Dans un autre écrit Derrida affirme: «Arraisonner, c'estdemander des papiers d'identité, une origine et une destination. C'est prétendre reconnaître un nom propre. Comment nommer sans arraisonner? Est-ce possible ?». Glas I. Que reste-t-il du savoir absolu ? (Paris: Denoël.Gonthier, 1981), 9.

17 Selon François Dosse, Althusser «s'efforce de sortir d'un marxisme officiel post-stalinien, d'un héritage enfermé dans le dogme. Il cherche à complexifier le marxisme, en croisant sa lecture avec des sciences humaines en plein essor. Il s'agit donc de repenser le marxisme en le sortant des scories des régimes qui s'en réclament». F. Dosse, Histoire du structuralisme. I. Le champ du signe, 1945-1966 (Paris: La Découverte, 1991), 359.

${ }^{18}$ J. Derrida, «Louis Althusser», Chaque fois unique la fin du monde (Paris: Galilée, 2003), 149.

${ }^{19}$ F. Gorog, «La mélancolie d'Althusser», La clinique lacanienne 1, Vol. 17 (2010): 111.
} 


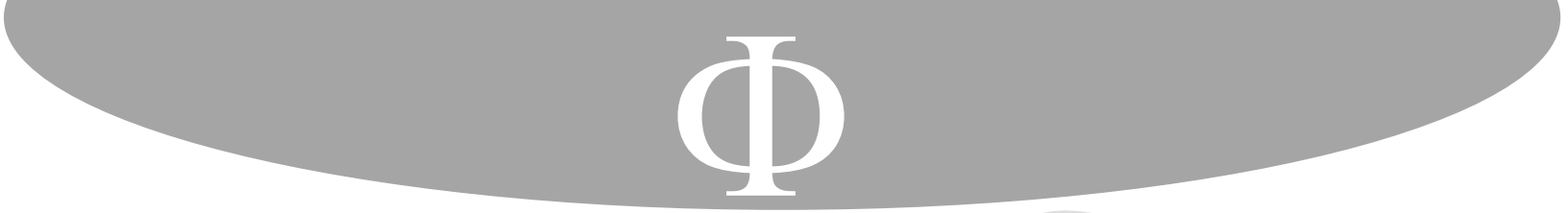

différentes de la question, Derrida, quant à lui, parlera du « crime » de sa circoncision ${ }^{54}$. On comprend, alors, peut-être mieux, sa critique de la métaphysique occidentale et ses constructions philosophiques qui lui paraissent dépendre de façon inconsidérée d'oppositions tranchées et de couples conceptuels irréductibles comme le spirituel et le matériel, l'universel et le particulier, l'éternel et le temporel, le masculin et le féminin ${ }^{55}$. Ces couples, en effet, posent un double problème. D'abord, de part leur rigidité extrême, tout ce qui ne s'inscrit pas parfaitement dans le rapport d'opposition se trouve marginalisé voire supprimé. Ensuite ces oppositions imposent un ordre hiérarchique. Ainsi, le réel de la circoncision mais aussi tout tranchement et retranchement prennent-ils une place centrale dans la pensée du philosophe.

Une scène touchant directement la question de la coupure ${ }^{56}$ occupe également la pensée d'Althusser ${ }^{57}$. Dans son autobiographie, le philosophe marxiste rappelle, en effet, avoir ressenti de profondes angoisses à cause d'un phimosis qui lui empoisonna la vie durant des années à Alger, mais aussi à Marseille. Le jeune Louis passait alors son temps à tirer sur la peau de mon sexe mais n'arrivait pas à en décalotter le gland. Il se pensait alors atteint d'une grave infection et ne parvenait pas à une érection complète et achevée dans l'éjaculation. Un jour sa mère alerta alors le père de Louis, et l'enferma littéralement avec l'enfant dans les toilettes. Durant plus d'une heure, dans le noir des toilettes, le père essaya alors de tirer sur la peau du prépuce de son fils.

Plus tard, à 30 ans, Louis n'aura encore jamais embrassé une femme. Lorsqu'un jour Hélène $^{58}$ - celle qui deviendra sa femme - prit l'initiative, un abîme d'angoisse s'ouvrit en lui «qui ne se referma plus» ${ }^{59}$. Au fond, pour Derrida, ce fut un «manque de peau» qui s'imposa

sexuelles, est alors refondée dans le symbolique». É. Marty, «Saint Paul et les Modernes. Universel symbolique, universel mimétique», Cités 1, Vol. 53 (2013): 179-180.

${ }^{54}$ «Chez les Juifs d'Algérie, on ne disait presque jamais la "circoncision" mais le "baptême", non la Bar Mitzwa mais "la communion", avec les conséquences de l'adoucissement, de l'affadissement, par acculturation apeurée, dont j'ai toujours souffert plus ou moins consciemment, d'événements innavouables, ressentis comme tels, pas "catholiques", violents, barbares, durs, "arabes", circoncision circoncise, accusation de meurtre rituel intériorisé, secrètement assumée». J. Derrida, Circonfession, 72.

${ }^{55}$ G. Borradori, «La déconstruction du concept de terrorisme chez Jacques Derrida», dans Le «concept» du 11 septembre, dialogues à New York (octobre-décembre 2001) avec Giovanna Borradori (Paris: Galilée, 2004), 198.

${ }^{56}$ En 1982, Althusser publiera un texte sur la «matérialisme de la rencontre». Or, selon Olivier Bloch, cet article «est peut-être à nouveau une façon de chercher une coupure. Cette obsession de la coupure caractérise la pensée d'Althusser. C'est pourquoi, dans son dernier texte, Althusser cherche une coupure avec son ou ses précédents matérialismes ». O. Bloch, « Face à Épicure et à la tradition matérialiste», A. W. Lasowski, Althusser et nous (Paris: Puf, 2016), 47.

${ }^{57}$ On pourrait d'ailleurs évoquer la difficulté du philosophe à couper avec le Parti comme le lui reprochera Jacques Rancière. Même lorsque sa compagne, Helène, s'en trouvera exclue, avec d'ailleurs son approbation officielle...

${ }^{58}$ Althusser aimera aussi d'autres femmes, comme la philosophe et traductrice Franca Madonia. Selon Élisabeth Roudinesco, «Louis Althusser tomba amoureux de Franca, découvrant à travers elle tout ce qui avait manqué dans son enfance et tout ce dont il était privé à Paris : une vraie famille, un art de vivre, une nouvelle façon de penser, de parler, de désirer». É. Roudinesco, Philosophes dans la tourmente (Paris: Fayard, 2005), 216.

${ }_{59}^{5}$ F. Gorog, «La mélancolie d'Althusser», 116. 


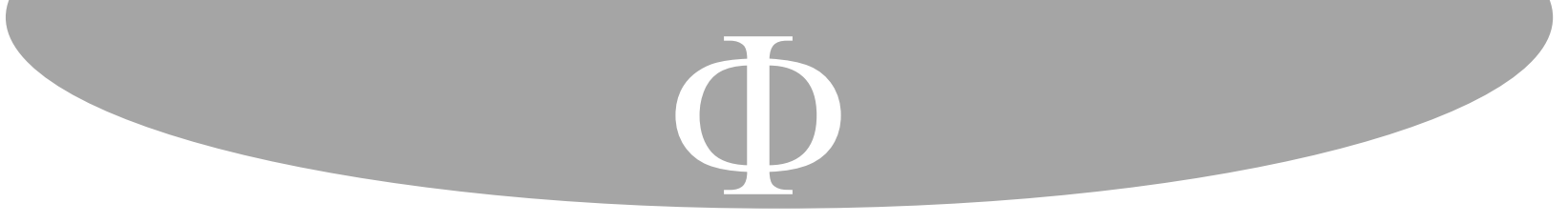

comme une blessure. Alors que pour Althusser, ce sera cette peau envahissante qui l' «étouffera» une partie de sa vie. Car le phénomène durera des années. Au point que notre philosophe restera longtemps convaincu de son anormalité. Comme s'il manquait à son anatomie quelque chose pour être un sexe d'homme. Ou bien comme si, en réalité, il ne disposait pas vraiment d'un sexe d'homme, ou qu'on l'en avait privé. L'auteur, d'ailleurs, n'hésite pas à trancher - si l'on peut dire - en déclarant : «Ma mère sans doute (...) m'avait littéralement "mis la main dessus" $\gg{ }^{60}$.

Le philosophe marxiste soulignera, par la suite, la nécessité, pour aimer, de ne pas être entamé dans l'intégrité de son corps et de son âme. Il déclarera même qu'il convient de ne pas être «châtré» - encore une fois, la coupure radiale - mais disposer de sa puissance d'être (Spinoza) sans en être «amputé d'une seule partie, sans être voué à le compenser sans l'illusoire ou le vide» ${ }^{61}$. Le phénomène se manifeste au niveau de la peur de la castration - la scène dans les toilettes - mais aussi de part la difficile coupure avec la mère. Notre auteur déclarera d'ailleurs avoir subi dans son corps ${ }^{62}$ et sa liberté, la loi des phobies de sa mère ${ }^{63}$. Il déclarera même qu'il n'existait que dans le désir de sa mère, jamais dans le sien, considéré comme inaccessible ${ }^{64}$. À d'autres moments, l'auteur de Lire le Capital, précisera qu'il faisait ce que sa mère voulait ; d'où sa volonté d'en rajouter pour se prouver, sur le dos des autres hommes, qu'il était bien un homme, doté d'un sexe et non cet être asexué que voulait sa mère ${ }^{65}$

La problématique de la coupure, sur le plan théorique cette fois, se retrouve donc aussi au sein de la philosophe d'Althusser mais à un autre niveau que dans la déconstruction. Rappelons, d'abord, à ce propos, que dans sa lecture de Marx, le philosophe reprit l'idée bachelardienne de «rupture épistémologique». Pour l'auteur de La formation de l'esprit scientifique, c'était en termes d'obstacle qu'il convenait de penser la connaissance

\footnotetext{
${ }^{60}$ L. Althusser, L'avenir dure longtemps, 128-129.

61 L. Althusser, L'avenir dure longtemps, 129. «La conjonction entre l'identification avec le caractère martyrisant de la mère et l'identification à un père de la mort sous les espèces de l'oncle Louis, d'un côté, et la profondeur de la relation à la mort que l'amour pathologique de la mère établit, d'un autre côté, font qu'Althusser ressent toute initiative de la mère comme dangereuse et angoissante, dans le sens où elle est porteuse d'une tentative de castration dans le réel. Par ailleurs, on retrouve aussi des pensées, des craintes de celui-ci de lui couper le prépuce dans le noir des toilettes lorsque le petit Louis souffrait de phimosis». G. Arce Ross, «L'homicide altruiste de Louis Althusser», 231.

${ }^{62}$ Le philosophe dira de façon radicale: «Je ne me sentais aucun corps» L. Althusser, L'avenir dure longtemps, 206.

${ }^{63}$ L. Althusser, L'avenir dure longtemps, 46. «Je vois bien maintenant que ma mère était littéralement assaillie de phobies : elle avait peur de tout, d'être en retard, peur de n'avoir plus (assez) d'argent, peut des courants d'air (elle avait toujours mal à la gorge, moi aussi, jusqu'à mon service militaire où je la quittai), une peur intense des microbes et de leur contagion, peur de la foule et de son bruit, peur des voisind, peur des accidents dans la rue et ailleurs, et par-dessus tout peur des mauvaises rencontres et des fréquentations douteuses qui peuvent mal tourner : disons-le, par dessus tout peur du sexe, du vol et du viol, c'est-à-dire peu d'être agressé dans son intégrité corporelle et d'y perdre l'intégrité problématique d'un corps encore morcelé». L. Althusser, $L$ 'avenir dure longtemps, 45.

${ }^{64} \mathrm{~L}$. Althusser, L'avenir dure longtemps, 53.

${ }^{65} \mathrm{~L}$. Althusser, L'avenir dure longtemps, 355.
} 


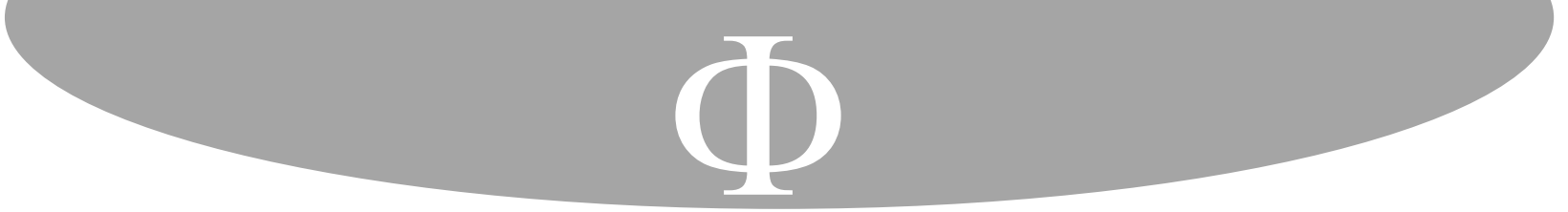

Lorsque le philosophe dit «l'impossible», cependant, il ne pense pas à l'obstacle, mais cherche plutôt à désigner cet autre régime du «possible-impossible» qu'il convient de penser en questionnant de toutes les manières - comme au sujet du don, du pardon, ou de l'hospitalité - en essayant de «déconstruire » l'héritage des concepts de «possibilité», de «pouvoir», d'impossibilité, etc ${ }^{83}$. Cet im-possible ne relève donc pas du privatif. Il ne s'agit pas de l'inaccessible. S'il n'arrivait que ce qui était déjà possible, donc anticipable et attendu, cela ne constituerait pas à proprement parler un événement. L'événement n'est possible, selon notre philosophe, que venu de l'impossible. Il arrive comme la venue de l'impossible, là où un «peut-être» nous prive de toute assurance et laisse l'avenir à l'avenir. «Ce "peutêtre" s'allie nécessairement à un "oui" : oui, oui à (ce) qui vient» ${ }^{84}$. La déconstruction, c'est l'impossible comme ce qui arrive.

Jacques Derrida ${ }^{85}$ déclare également qu'«il n’y a pas de hors-texte». Une telle formule pourrait laisser croire que le philosophe se contente de déplier une position structuraliste. Il s'agirait, alors, simplement d'attester le principe d'immanence ou la clôture du signe. Or, pour notre auteur, cette expression signifie, au contraire qu'il n'y a pas de hors contexte $^{86}$. Le vocable renvoie, alors, à toute l'histoire-réelle-du-monde dans laquelle la valeur d'objectivité et même, plus largement, celle de vérité ont pris un sens et se sont imposées. Ce qui, du reste, ne les discrédite en rien. Derrida comprend parfois la déconstruction comme la prise en compte de ce contexte sans bord, l'attention la plus vive et la plus large possible au contexte. Il s'agit, par conséquent, d'un mouvement incessant de recontextualisation. Le philosophe utilise l'expression «histoire-réelle-du-monde», pour souligner que le concept de texte ou de contexte qui le guide comprend le monde, la réalité et l'histoire. Le «texte», ici, ne renvoie pas au livre, à cet objet que l'on trouve dans une bibliothèque. Il ne suspend donc pas la référence à l'histoire, au monde, à la réalité, à l'être ou à l'autre ${ }^{87}$.

Plus fondamentalement, l'auteur de Limited Inc remet en cause l'idée d'une expérience pure. Pour lui, «la res, (...) n'est pas seulement l'objet, la chose mais tout ce que notre tradition a baptisé comme le réel : le réel historique, économique, politique, sexuel et ainsi de suite. (...) la réalité n'est pas plus dans le texte qu'elle n'est la référence rassurante du hors-texte. Troisième voie comme toujours : le lexique de la prétendue "réalité" et sa

\footnotetext{
${ }^{83} \mathrm{~J}$. Derrida, «Auto-immunité, suicides réels et symboliques», Le «concept» du 11 septembre, 178.

${ }^{84}$ J. Derrida, «Comme si c'était possible, Within such limits», Revue Internationale de Philosophie 3, Vol. 205 (1998): 497-529.

${ }^{85}$ Dans les limites de cet article, nous ne pouvons développer la critique derridienne de la pensée lacanienne, disséminée dans un certain nombre d'écrits dont: La carte postale. De Socrate à Freud et au-delà (Paris: Flammarion, 1980).

${ }^{86}$ J. Derrida, Limited Inc., Présentation et traduction par Elisabeth Weber (Paris: Galilée, 1990), 252.

${ }^{87}$ Par ailleurs, selon Derrida, l'univers de la signification n'est pas autonome, indépendant, «il ne cesse de déborder sur le dehors de lui-même, non pas au titre de la référence, comme on l'admet volontiers dans la vision empiriste, mais au titre de l'événementialité de la parole», J-.M. Salanskis, «La philosophie de jacques Derrida et la spécificité de la déconstruction au sein des philosophies du linguistic turn», Coordonné par C. Ramond, Derrida : la déconstruction (Paris: Puf, 2005), 31.
} 


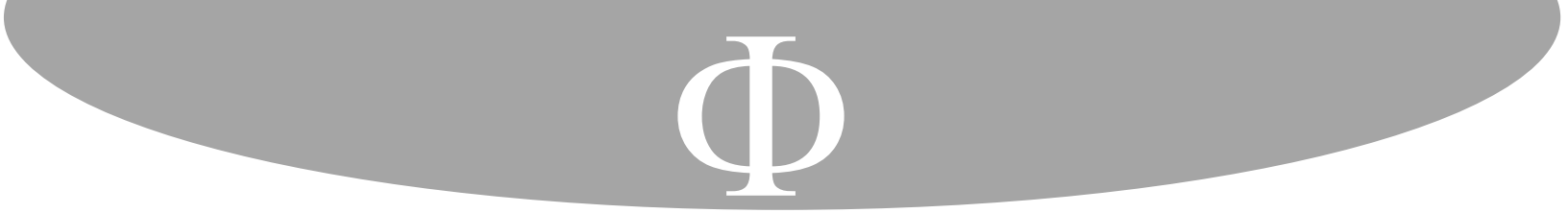

Derrida, Jacques. Sauf le nom. Paris: Galilée, 1993.

Derrida, Jacques. Stiegler, Bernard. Échographie de la télévision, entretiens filmés. Paris: Galilée, 1996.

Derrida, Jacques. «Comme si c'était possible, Within such limits». Revue Internationale de Philosophie 3, Vol. 205 (1998): 497-529.

Derrida, Jacques. E. Roudinesco. De quoi demain... Dialogue. Paris: Fayard/Galilée, 2001.

Derrida, Jacques. Marx \& Sons, Actuel Marx. Paris: Puf/Galilée, 2002.

Derrida, Jacques. «Louis Althusser». Chaque fois unique la fin du monde. Paris: Galilée, 2003.

Derrida, Jacques. Judéités. Questions pour Jacques Derrida. Paris: Galilée, 2003.

Derrida, Jacques. Circonfession. En Jacques Derrida par G. Bennington et J. Derrida. Paris: Seuil, 2008.

Dosse, François. Histoire du structuralisme. I. Le champ du signe, 1945-1966. Paris: La Découverte, 1991.

Freud, Sigmund. L'homme Moïse et la religion monothéiste. Traduit de l'allemand par Anne Berman. Paris: Gallimard, 1948.

Freud, Sigmund. Ma vie et la psychanalyse (1925), suivi de Psychanalyse et médecine, traduit de l'allemand par Marie Bonaparte. Paris: Gallimard, 1950.

Goldstick, Daniel. «Lecture d'Althusser». Philosophiques 2, Vol 12 (1985): 363-381.

Gorog, François. «La mélancolie d'Althusser». La clinique lacanienne 1, Vol. 17 (2010): 109-126.

Kojève, Alexandre. Introduction à la lecture de Hegel. Paris: Gallimard, 1947.

Lacan, Jacques. Séminaire XIV, La logique du fantasme, séance du 10 mai 1967, 170. Consulté le 11 avril 2021. http://staferla.free.fr/S14/S14\%20LOGIQUE.pdf.

Lacan, Jacques. Le séminaire. Livre XI. Les quatre concepts fondamentaux de la psychanalyse. Texte établi par jacques-Alain Millier. Paris: Seuil, 1973.

Lindner, Urs. «Repenser la ''coupure épistémologique'. Lire Marx avec et contre Althusser». Actuel Marx 1, Vol. 49 (2011): 121-139.

Macherey, Pierre. Le sujet des normes. Paris: Amsterdam, 2014.

Marty, Éric. «Saint Paul et les Modernes. Universel symbolique, universel mimétique». Cités 1, Vol. 53 (2013): 167-186.

Marx, Karl, Engels, Frédérich. Manifeste du Parti communiste, 1948. Introduction, note et commentaire de F. Châtelet, traduction de C. Lyotard. Paris: Livre de poche, 1973.

Nietzsche, Frédéric. Le gai savoir. Traduit de l'allemand par Alexandre Vialatte. Paris: Gallimard, 1950.

Major, René. Lacan avec Derrida. Analyse désistantielle. Paris: Flammarion, 2001.

Major, René. «Derrida, lecteur de Freud et de lacan». Études françaises 1-2, Vol. 38 (2002): 165-178.

Mauss, Marcel. Sociologie et anthropologie. Paris: Puf, 1950.

Pommier, Gérard. La mélancolie. Vie et æuvre d'Althusser. Paris: Flammarion, 2009.

Peeters, Benoît. Derrida. Paris: Flammarion, 2010.

Peeters, Benoît. «Althusser et Derrida, politique et affection». Consulté le 11 avril 2021, https://www.imec-archives.com/papiers/benoit-peeters/.

Poché, Fred. Penser avec Jacques Derrida. Comprendre la déconstruction. Lyon : Chronique sociale, 2007. 


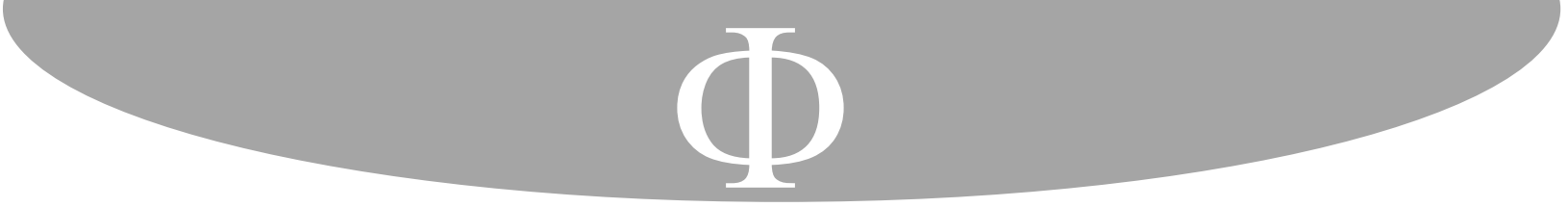

Ramond, Charles. «Matérialisme et hantologie». Cités 30 (2007): 53-63.

Rimbaud, Arthur. Poésies. Paris: Librairie Générale Française.

Roudinesco, Élisabeth. Histoire de la psychanalyse en France. 2, 1925-1985. Paris: Seuil, 1986.

Roudinesco, Élisabeth. Philosophes dans la tourmente. Paris: Fayard, 2005.

Roudinesco, Élisabeth. Jacques Lacan. Esquisse d'une vie, histoire d'un système de pensée. Paris: Fayard, 1993.

Salanskis, Jean-Michel. «La philosophie de Jacques Derrida et la spécificité de la déconstruction au sein des philosophies du linguistic turn». Coordonné par $\mathrm{C}$. Ramond. Derrida: la déconstruction. Paris: Puf, 2005.

Sartre, Jean-Paul. L'idiot de la famille. Guastave Flaubert de 1821 à 1857. Paris: Gallimard,1988.

Simon, Michel. Comprendre les idéologies. Les croyances,-les idées - les valeurs. Lyon: Chronique Sociale, 1978.

Vergani, Mario. «Les chances du réel: Derrida et Lucrèce». En A. Gigandet, Lucrèce et la modernité: le vingtième siècle. Paris: Armand Colin, 2013.

Enviado: 11 de mayo de 2021

Aceptado: 19 de julio de 2021 